Proc. Indian Acad. Sci. (Earth Planet. Sci.), Vol. 100, No. 3, September 1991, pp. 219-233.

(C) Printed in India.

\title{
Atmospheric surface layer characteristics with MONTBLEX data
}

\author{
P BANDYOPADHYAY, S CHOWDHURY and \\ G BANDYOPADHYAY \\ Department of Aerospace Engineering, Indian Institute of Technology, Kharagpur 721302 , \\ India \\ MS received 27 November 1990; revised 8 July 1991
}

\begin{abstract}
Data obtained from the $30 \mathrm{~m}$ high MONTBLEX tower installed at the Indian Institute of Technology, Kharagpur are described. Data on wind speed and direction, temperature and humidity were recorded during the first week of July 1989 at six levels on the tower. They indicate some of the atmospheric surface layer characteristics. Using two levels of tower data involving wind speed and temperature a profile method was used for computing the surface fluxes of heat and momentum. A method for calculating the surface roughness length using the data was also used to obtain the vertical windspeed profile. The computed wind profile was compared with observations, while the surface roughness length was compared with values obtained by a least square fit. The computed surface fluxes were compared with theoretical values obtained by an energy budget method which uses only wind data at one level. The agreement was reasonably good.
\end{abstract}

Keywords. MONTBLEX; surface layer; stability; inversion; surface fluxes: wind profile.

\section{Introduction}

A $30 \mathrm{~m}$ high tower was installed at the Indian Institute of Technology, Kharagpur $\left(87^{\circ} 20^{\prime} \mathrm{E}, 20^{\circ} 20^{\prime} \mathrm{N}\right)$ in July 1988 as part of the MONTBLEX (MONsoon Trough Boundary Layer Experiment) programme. The tower was equipped with fast response instruments to measure the wind speed, wind direction, temperature and humidity at six levels: $1,2,4,8,15$ and $30 \mathrm{~m}$. It was set up at the farmlands near the southeastern boundary of the Institute (figure 1). The relief of the test region is relatively flat (a slope of $10^{-2}$ over $400 \mathrm{~km}^{2}$ ). The tower site enjoys an uninterrupted fetch of $500 \mathrm{~m}$ towards the south, the direction of summer monsoon wind. As the region has a flat and fairly open terrain the influence of topography and ground obstacles on wind characteristics is expected to be small.

A pilot experiment was conducted during the first week of July, 1989 and data on the vector wind, temperature and humidity were collected at specific times of the day and recorded on the hard disc of a PC-XT. However, surface fluxes of heat and momentum, which determine the state of the atmospheric surface layer, were not measured.

In this paper, we analyse the tower data to obtain the surface layer characteristics. To estimate the surface fluxes theoretically, two levels of tower data were used. An attempt was made to calculate the surface roughness length $\left(Z_{0}\right)$, and thereby the vertical wind speed profile. 


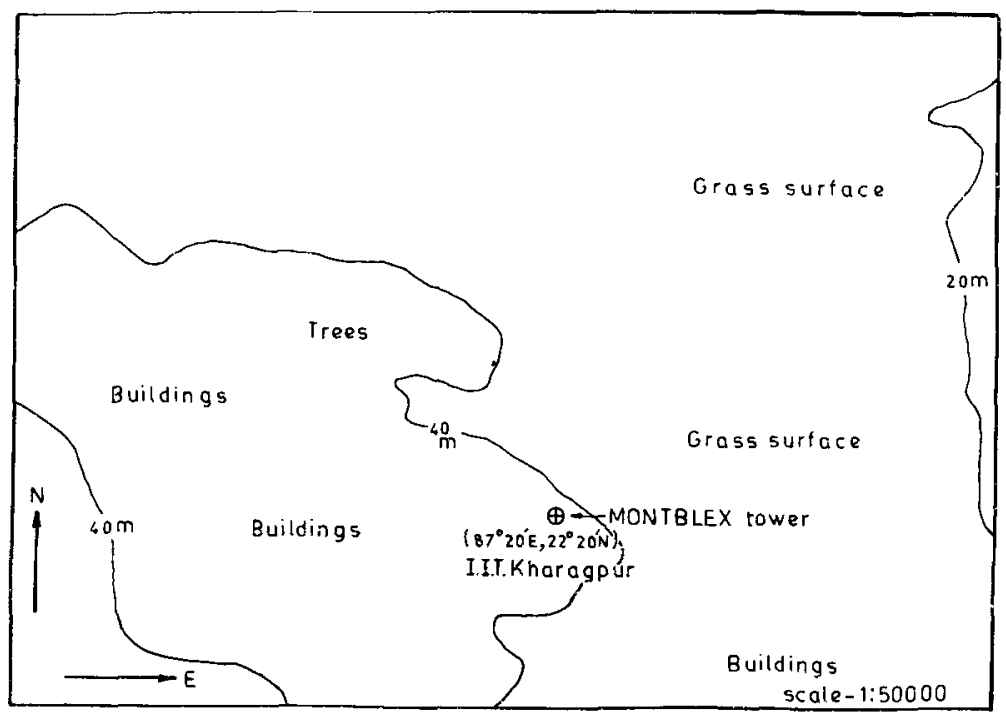

Figure 1. Relief map of the test region.

\section{Data representation and analysis}

Data on the vector wind, temperature and humidity were recorded at specific times every day for the first week in July 1989 for 10-min duration on a fast response data acquisition system. The sampling rate was 8.42 samples/s. For each 10 -min duration, 5052 readings were recorded for each measured quantity at six leyels. This amounts to a total of approximately one megabyte of computer memory for each observation at a particular time.

Data were recorded on a PC-XT and subsequently compiled on a CYBER 180 system. A program was developed for calculation of mean values for each measured quantity at a specified time. Before calculating mean values, the quality of the data was checked by comparing two consecutive values. The obviously erroneous data were removed and compiled as not recorded. Mean values were then calculated by averaging over 10 -min intervals.

The mean wind speeds obtained by the above procedure are shown in figure 2 at heights of $1.312 \mathrm{~m}, 8.312 \mathrm{~m}$ and $30.312 \mathrm{~m}(31.2 \mathrm{~cm}$ is the height at which the sensor was located at each of six levels). This figure illustrates the diurnal variation over 48 hours.

The vertical variation of mean temperature is shown in figure 3 for 2 July 1989 . The vertical profile is characterized by a low-level inversion during the whole day. The temperature stratification indicates typical stability characteristics of the surface layer. In general the surface layer seems to be stable up to $4 \mathrm{~m}$, unstable between $4 \mathrm{~m}$ and $15 \mathrm{~m}$ and near-neutral up to $30 \mathrm{~m}$. The vertical profiles of temperature indicating these typical characteristics were also observed on subsequent days.

The typical stability characteristics are illustrated in figure 4 . The microscale turbulence induced is weak and intermittent up to $4 \mathrm{~m}$ which indicates the damping effect of a stable stratification on turbulence, particularly at $1 \mathrm{~m}$ and $4 \mathrm{~m}$. Between 4 and $15 \mathrm{~m}$, instability of the surface layer contributes to development of mechanical 


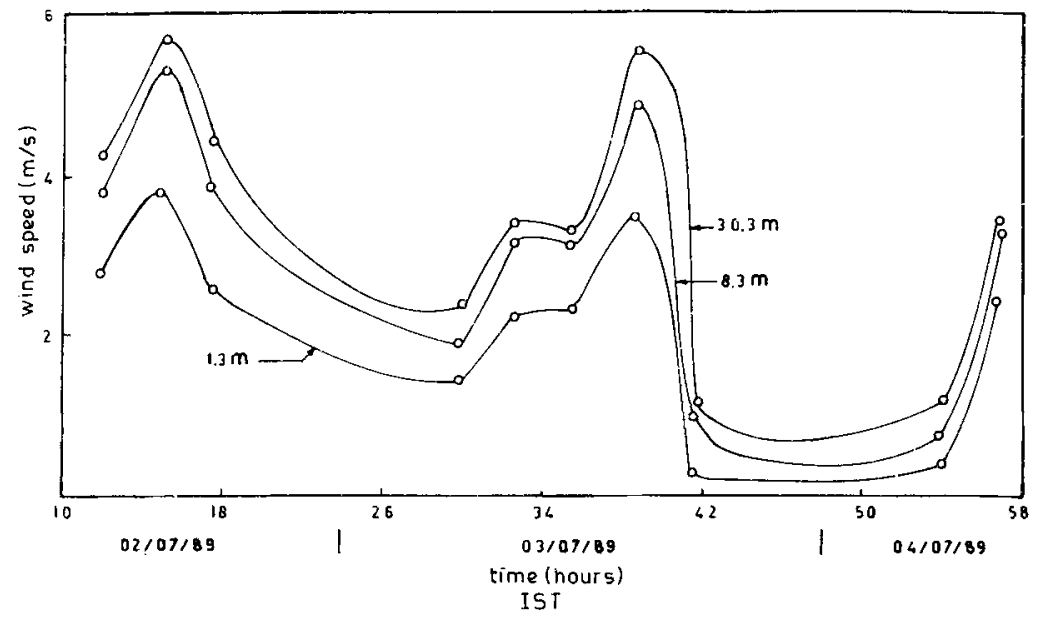

Figure 2. Diurnal variation of different levels over 48 hours.

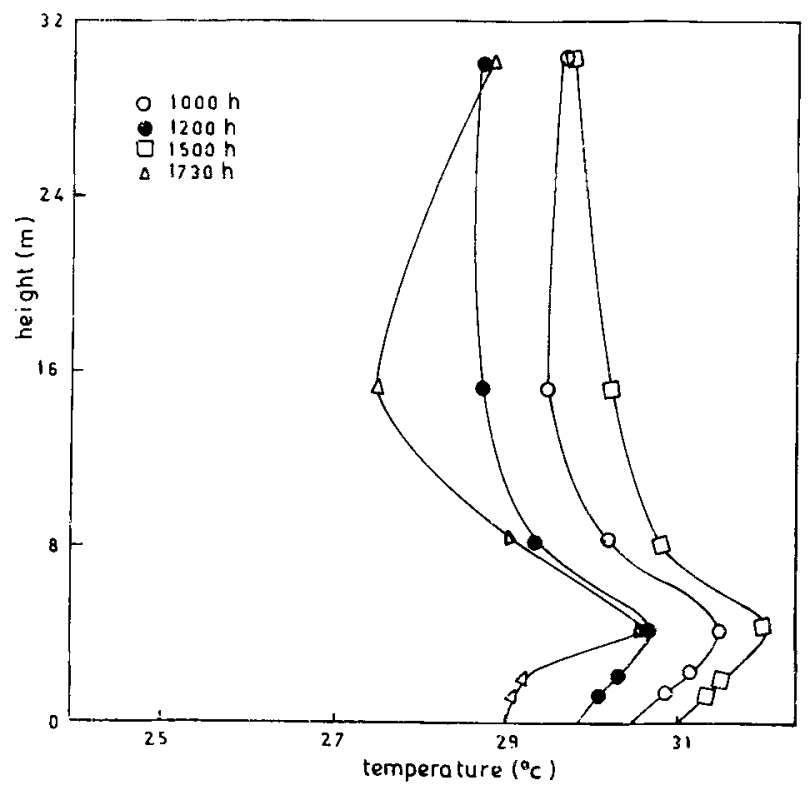

Figure 3. Vertical temperature profile at different hours on 2 July 1989.

turbulence. At $30 \mathrm{~m}$, turbulence is again suppressed indicating the small effect of ground surface irregularities at this height.

\section{Calculation of fluxes and wind speed profile using profile method}

\subsection{Surface fluxes of heat and momentum}

The surface fluxes of heat and momentum were obtained from the observed profiles of wind and temperature using the Monin-Obhukhov similarity theory. The 


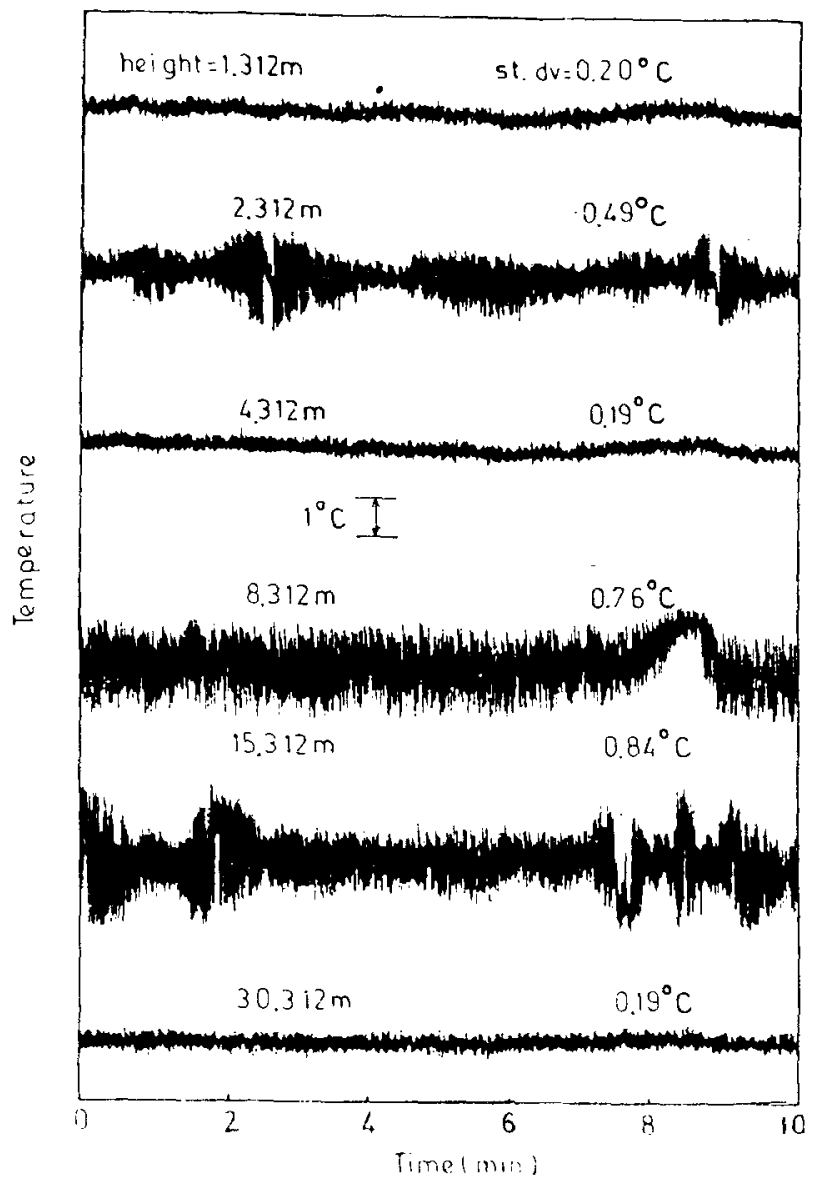

Figure 4. Simultaneous time series of temperature at six levels above the ground (1000h, 2 July 1989).

Monin-Obhukhov length $(L)$ is given by

$$
L=\mathrm{T} u_{*}^{2} / k g \theta_{*},
$$

where $T$ is the absolute temperature of air, $g$ the acceleration due to gravity, $k$ is the von Karman constant $(0 \cdot 41), u_{*}$, the frictional velocity and $\theta_{*}$ the temperature scale.

Following Dyer (1974) and Paulson (1970), a generalized expression for wind speed at any height $z$ is given by

$$
u(z)=\left(u_{*} / k\right)\left[\ln \left(Z / Z_{0}\right)-\psi_{M}(z / L)+\psi_{M}\left(z_{0} / L\right)\right]
$$

and the potential temperature may be expressed by

$$
\theta(z)=\left(\theta_{*} / k\right)\left[\ln \left(z / z_{0}\right)-\psi_{H}(z / L)+\psi_{H}\left(z_{0} / L\right)\right],
$$

where $z_{0}$ is the surface roughness length and $\psi_{M}$ and $\psi_{H}$ are stability functions.

The stability functions for unstable $(L<0)$ and stable $(L>0)$ conditions are (Paulson 1970; Businger et al 1971; Dyer 1974). 
For $L<0$

$$
\begin{aligned}
& \psi_{M}=2 \ln \left[\frac{1}{2}(1+x)\right]+\ln \left[\frac{1}{2}\left(1+x^{2}\right)\right]-2 \tan ^{-1}(x)+(\pi / 2), \\
& \psi_{H}=2 \ln \left[\frac{1}{4}\left(1+x^{2}\right)\right]
\end{aligned}
$$

where

$$
x=[1-16(z / L)]^{1 / 4} .
$$

For $L>0$

$$
\begin{aligned}
& \psi_{M}=-5 z / L \\
& \psi_{H}=-0.7 z / L-[0.75 z / L-10 \cdot 72] \exp [-0.35 z / L]-10.72 .
\end{aligned}
$$

These functions are valid for $z_{0} \ll z<L$. It appears, however, that in unstable conditions (2) in combination with (4) and (6) can be used at heights where $z \gg L$ (Holstag 1984).

In stable conditions $(L>0),(2)$ in combination with (7) fails for $z>L$. Equation (7) can, however, be replaced by another empirical function (van Ulden and Holstag 1985 ) that has the same performance for $z<L$, but a much better performance for $z>L$. This function is

$$
\psi_{M}=-17[1-\exp (-0.29 z / L] .
$$

The unknown are $u_{*}, \theta_{*}, L, z_{0}$ and $T$. Once these quantities are obtained, the flux of momentum $\tau$ can be obtained from

$$
\tau=\rho u_{*}^{2}
$$

and the flux of sensible heat $H$ from the expression

$$
H=-\rho C_{p} u_{*} \theta_{*}
$$

where $\rho$ is the density of air and $C_{P}$ is the specific heat at constant pressure.

A number of methods have been developed for calculating surface fluxes of heat and momentum from observed wind data. If more than two levels of wind and temperature data are available, a least square technique is commonly employed (Paulson 1970; Businger et al 1971; Nieuwstadt 1978) for calculating $u_{*}$ and $\theta_{*}$.

When wind and temperature data are available at two levels, $u_{*}$ and $\theta_{*}$ are solved by iteration. This is referred to as the profile method (Nieuwstadt 1978; McBean 1979; Berkowicz and Prahm 1982).

If wind and temperature data are available at only one level, an energy budget method developed by van Ulden and Holstag (1985) may be used for getting an estimate of $u_{*}$ and $\theta_{*}$.

A simplified profile method is presented here for computing the surface fluxes of heat and momentum. The method requires input of wind speed at two levels $\left(z_{1}\right.$ and $\left.z_{2}\right)$, temperature at two leveis $\left(z_{3}\right.$ and $\left.z_{1}\right)$ and $T \cdot u_{*}$ and $\theta_{*}$ are then obtained by satisfying (2) and (3) at two levels and subtracting one from the other. We have

$$
\begin{aligned}
& u_{*}=k\left[u\left(z_{2}\right)-u\left(z_{1}\right)\right]\left[\ln \left(z_{2} / z_{1}\right)-\psi_{M}\left(z_{2} / L\right)+\psi_{M}\left(z_{1} / L\right)\right]^{-1}, \\
& \theta_{*}=k\left[\theta\left(z_{4}\right)-\theta\left(z_{3}\right)\right]\left[\ln \left(z_{4} / z_{3}\right)-\psi_{H}\left(z_{1} / L\right)+\psi_{H}\left(z_{3} / L\right)\right]^{-1} .
\end{aligned}
$$


The advantage of this procedure is that in calculating $u_{*}$ and $\theta_{*}$ from (12) and (13) an estimate of $z_{0}$ is not needed.

Once the equations are set up, $u_{*}, \theta_{*}$ and $L$ can be obtained by an iterative procedure. The initial values of $u_{*}$ and $\theta_{*}$ can be obtained from (12) and (13) by assuming a neutral wind profile (i.e., by putting $\psi_{M}=\psi_{H}=0$ ). Using (1) $L$. is then computed. The new value of $L$ is then substituted in (12) and (13) to obtain improved values for $u_{*}$ and $\theta_{*}$ and this cycle is repeated till convergence is achieved. Once convergence is achieved, the surface fluxes of heat and momentum can be obtained from (10) and (11).

\subsection{Surface roughness length}

Generally the surface roughness length $\left(z_{0}\right)$ is obtained from wind measurements by a method given by Wieringa $(1976,1980,1983)$. This relates $z_{0}$ either to the normalized standard deviation of wind speed or to the ratio of averaged wind speed observed in a given period, and the maximum gust recorded during the same period. Alternatively, when no gust records are available, an approximate value of the roughness length can be obtained from visual terrain description together with the roughness classification of Davenport (1960). This has been described by Wieringa (1980). Surface roughness length can be calculated from the observed wind data at more than two levels by a least square technique (Paulson 1970).

A method for calculating $z_{0}$ in unstable stratification $(L<0)$ from observed wind data at one level is presented here. Once $u_{*}, \theta_{*}$ and $L$ are obtained, $z_{0}$ can be obtained from (2) using observed wind data at any one level. The procedure is as follows. Equation (2) may be expressed by

or,

$$
u(z)=\frac{u_{*}}{k} \ln \frac{-16 \mathrm{z} / L}{-16 z_{0} / L}-\psi_{M}(z / L)+\psi_{M}\left(z_{0} / L\right),
$$

$$
\psi_{M}\left(z_{0} / L\right)-\ln \left(-16 z_{0} / L\right)=\left(k u(z) / u_{*}\right)-\ln (-16 z / L)+\psi_{M}(z / L) .
$$

The right side of (14) can be evaluated from observed wind speed $u(z)$ at any level $z$. Terms containing $z_{0}$ on the left side of the equation can be expanded by applying (6) and (4) at $z=z_{0}$.

Applying (6) at $z=z_{0}$ gives us

or,

$$
x_{0}=\left[1-16\left(z_{0} / L\right)\right]^{1 / 4}
$$

$$
-16\left(z_{0} / L\right)=x_{0}^{1}-1
$$

From (4) at $z=z_{0}$ we have

$$
\begin{aligned}
\psi_{M}=\left(z_{0} / L\right) & =2 \ln \left[\frac{1}{2}\left(1+x_{0}\right)\right]+\ln \left[\frac{1}{2}\left(1+x_{0}^{2}\right)\right]-2 \tan ^{-1}\left(x_{0}\right)+(\pi / 2) \\
& =2 \ln \left(1+x_{0}\right)+\ln \left(1+x_{0}^{2}\right)-\ln 8-2 \tan ^{-1}\left(x_{0}\right)+(\pi / 2) .
\end{aligned}
$$

Using (15) and (16), (14) can be written as

$$
\begin{aligned}
& 2 \ln \left(1+x_{0}\right)+\ln \left(1+x_{0}^{2}\right)-\ln 8-2 \tan ^{-1}\left(x_{0}\right)+\pi / 2-\ln \left(x_{0}^{4}-1\right) \\
& =\left(k u(z) / u_{*}\right)-\ln (-16 z / L)+\psi_{M}(z / L)
\end{aligned}
$$


or

$$
\begin{aligned}
& 2 \ln \left(1+x_{0}\right)-\ln \left(x_{0}^{2}-1\right)-2 \tan ^{-1}\left(x_{0}\right) \\
& \quad=\left(k u(z) / u_{*}\right)-\ln (-16 z / L)+\psi_{M}(z / L)+\ln 8-(\pi / 2) .
\end{aligned}
$$

This can be expressed by

where

$$
f\left(x_{0}\right)=2 \ln \left(1+x_{0}\right)-\ln \left(x_{0}^{2}-1\right)-2 \tan ^{-1}\left(x_{0}\right)-b=0
$$

$$
b=\left(k u(z) / u_{*}\right)-\ln (-16 z / L)+\psi_{M}(z / L)+\ln 8-(\pi / 2) .
$$

$b$, given by (18) is known from the observed wind speed $u(z)$ at any level $z$. Equation (17) can now be solved by a first-order Newton-Raphson method to find $x_{0}$ corresponding to a surface roughness length $z_{0}$. Convergence of the iterative scheme depends on the initial value of $x_{0}$. The initial value of $x_{0}$ can be obtained from Davenport's (1960) terrain classification. Once the solution for $x_{0}$ converges, $z_{0}$ can be obtained from (15).

\subsection{Vertical wind speed profile}

Once $u_{*}$ and $z_{0}$ are calculated using the procedures outlined in $\$ 3 \cdot 1$ and $3 \cdot 2$, the vertical wind speed profile for an unstable stratification can be obtained from (2), i.e.

$$
u(z)=\left(u_{*} / K\right)\left[\ln \left(z / z_{0}\right)-\psi_{M}(z / L)+\psi_{M}\left(z_{0} / L\right)\right],
$$

where $\psi_{M}$ is given by (4).

As $z_{0}$ cannot be calculated for a stable stratification $(L>0)$, the vertical wind speed profile may be obtained by satisfying (2) at two levels and subtracting one from the other. We have

$$
u(z)=u\left(z_{1}\right)+\left(u_{*} / K\right)\left[\ln \left(z / z_{1}\right)-\psi_{M}(z / L)+\psi_{M}\left(z_{1} / L\right)\right],
$$

where $u\left(z_{1}\right)$ is the observed wind speed at level $z_{1}$.

\section{Calculation of fluxes using least squares}

Following Paulson (1970) a least square method is available for calculating $z_{0}, \omega_{*}$ and $\theta_{*}$ and the fluxes. In this approach we assume that at $z=z_{0}$

since

$$
\psi_{M}\left(z_{0} / L\right) \approx 0
$$

$$
x_{0}=\left[1-16 z_{0} / L\right]^{1 / 4} \approx 1
$$

With this assumption (2) can be written as

$$
u(z)=\left(u_{*} / k\right)\left[\ln \left(z / z_{0}\right)-\psi_{M}(z / L)\right] .
$$

Rearranging terms we have

$$
\ln z-\psi_{M}(z / L)=\left(k / u_{*}\right) u(z)+\ln z_{0} .
$$


This is a considerable simplification as $z_{0}$ appears only once in (22). It avoids the need to specify $k$ unless an actual value of $u_{*}$ is needed.

A straight line can now be fitted by least squares to wind speed observations plotted against $\ln z-\psi_{M}(z / L)$. The ordinate intercept of the straight line yields $z_{0} \cdot u_{*}$ may be obtained from the slope of the straight line by taking $k$, von Karman's constant to be $0 \cdot 41$.

A similar procedure may be used for obtaining $\theta_{*}$ from temperature observations.

It may be noted here that for neutral conditions

$$
\psi_{M}(z / L)=\psi_{M}\left(z_{0} / L\right)=0 .
$$

Consequently, the observed wind speed profile will be linear on a logarithmic plot and a least square fit is not needed for calculating $z_{0}$.

\section{Calculation of fluxes with the energy budget method}

As the surface fluxes of heat the momentum computed by the present method will be compared with the energy budget method, a brief description of this method is presented. The method developed by van Ulden and Holstag (1985) uses only one level of wind observations, surface roughness length and air temperature.

In the absence of vertical temperature data, equation (13) is not used for calculating $\theta_{*}$. This is replaced by information on the surface energy budget

$$
H+\lambda E=Q^{*}-G
$$

where $\lambda E$ is the latent heat flux ( $\lambda$ is the latent heat of vaporization and $E$ is the evaporation), $Q^{*}$ is the net radiation and $G$ the soil heat flux. Using $H=-\rho C_{P} u_{*} \theta_{*}$, $(23)$ is

$$
\theta_{*}=\frac{\lambda E-Q^{*}+G}{\rho C_{p} u_{*}} .
$$

$\lambda E, Q^{*}$ and $G$ are parametrized in terms of total cloud cover $N$, the solar elevation $\phi$, air temperature $T$, the friction velocity $u_{*}$ and $\theta_{*}$. Thus, $\theta_{*}$ is given by an expression of the following type

$$
\theta_{*}=f\left(H, \phi, T, u_{*}\right)
$$

Equation (25) is now used instead of (13). The subsequent procedure of finding $u_{*}$ and $\theta_{*}$ from (2) and (25) by iteration is similar to what was used earlier.

The details of modelling $\lambda E, Q^{*}$ and $G$ are given by Holstag and van Ulden (1983) and van Ulden and Holstag (1985). A short description is given here for continuity.

\subsection{The net radiation}

The net radiation $Q^{*}$ consists of (i) shortwave solar radiation $K^{*}$ and (ii) the difference between the outgoing radiation $L^{-}$from the earth's surface and the incoming radiation 
$L^{+}$from the atmosphere. Thus

$$
Q^{*}=K^{*}+L^{+}-L^{-}
$$

The net shortwave radiation is parametrized by

$$
K^{*}=\left(a_{1} \sin \phi+a_{2}\right)\left(1-b_{1} N^{b_{2}}\right)(1-r)
$$

where $\left(a_{1} \sin \phi+a_{2}\right)$ is the incoming solar radiation with clear skies and $a_{1}$ and $a_{2}$ are the empirical constants. The reduction factor $\left(1-b_{1} N^{b_{2}}\right)$ gives the interception of solar radiation by clouds with $N$ the fraction of sky covered by clouds and $b_{1} \cdot b_{2}$ are empirical constants. The reduction factor $(1-r)$ is due to the reflection of incoming solar radiaton at the surface, where $r$ is the albedo. Typical values of the quantities are $a_{1}=990 \mathrm{Wm}^{-2}, a_{2}=-30 \mathrm{~W}, b_{1}=0.75, b_{2}=3.4$ and for grass surface $r=0.23$.

The incoming longwave radiation is parametrized by

$$
L^{+}=C_{1} \sigma T_{r}^{6}+C_{2} N
$$

and the outgoing longwave radiation from earth's surface

$$
L^{-}=\sigma T_{0}^{1}
$$

Here $\sigma=5.67 \times 10^{-8} \mathrm{Wm}^{-2} \mathrm{~K}^{-4}$ is the Stefen-Boltzmann constant, $T_{r}$ the air temperature at a reference height, $C_{1}=9.35 \times 10^{-6} \mathrm{~K}^{-2}$ and $C_{2}=60 \mathrm{Wm}^{-2}$ are einpirical coefficients and $T_{0}$ is the temperature at the surface. If the atmospheric surface layer is assumed to be isothermal $T_{r}$ is taken as $T_{0}$; otherwise, a correction factor (van Ulden and Holstag 1985) can be used for obtaining $T_{r}$ from $T_{0}$.

\subsection{The soil heat flux}

The soil heat flux $\mathrm{G}$ is small compared with $Q^{*}$ for land during daytime. A good estimate for $G$ is given by

$$
G=C_{G} Q^{*}
$$

where $C_{G}=0 \cdot 1$ for a grass covered surface.

\subsection{Evaporation}

The evaporation $\lambda E$ is formally given by the Penman-Monteith approach, which has been considerably simplified by Holstag and van Ulden (1983). In its simplified form it is

$$
\lambda E=\frac{\alpha}{(1+\gamma / s)}\left(Q^{*}-G\right)+\beta .
$$

Here $\gamma=C_{P} / \lambda$, where $C_{P}$ is the specific heat of air at constant pressure. $\alpha$ and $\beta$ are empirical factors. The ratio $\gamma / s$ are tabulated as a function of temperature at standard pressure by Holstag and van Ulden (1983). At temperatures other than the values 
given in a tabular form $\gamma / \mathrm{s}$ can be obtained by interpolation. For a grass-covered surface, $\alpha$ and $\beta$ are $\alpha=1$ and $\beta=20 \mathrm{Wm}^{-2}$.

\section{Computational details}

A computer program was developed in FORTRAN IV based on a two-level wind and temperature data described in $\S 3$. MONTBLEX data are available at six levels. Numerical experimentation with different combinations shows that the best fit with observed data is obtained when two levels are over a large height interval. This is probably because of the typical temperature profile. For all calculations, $z_{1}=z_{3}=1.312 \mathrm{~m}$ and $z_{2}=z_{4}=30 \cdot 312 \mathrm{~m}$ was assumed.

The iterative scheme for calculating the Monin-Obukhov length $L$, described in $\S 3 \cdot 1$, was repeated till successive values of $L$ did not change by more than $1 \%$. We found that only a few cycles were needed (usually not more than 5) to achieve the desired accuracy.

The Newton-Raphson iteration scheme for calculating $z_{0}$, outlined in $\S 3 \cdot 2$, usually converges within ten iterations to $1 \%$ accuracy provided the initial values was chosen properly. In the present calculations, the observed wind speed at $1.312 \mathrm{~m}$ was used. The initial value of $z_{0}$ for the MONTBLEX tower site in an open flat terrain was assumed to be $0.03 \mathrm{~m}$ and the corresponding initial value of $x_{0}$, using equation (15) was taken as 1.005 for an unstable stratification.

Another computer program was developed in FORTRAN IV for calculating $z_{0}$ using a least square fit for the observed wind data at six levels plotted against $\ln z-\psi_{M}(z / L)$. The input data for $\ln z-\psi_{M}(z / L)$ was obtained as the output of the program based on profile measurements. The program was also used for calculating $\theta_{*}$ from observed temperature data. However, in this case the scatter was high and a linear plot was not meaningful.

To compare the results obtained by the present method, results were also obtained by an energy budget method using the FORTRAN package developed at KNMI (Koninklijk Nederlands Meteorologish Istitut), the Netherlands. The package contains several subroutines for different inputs. For the present comparison, the routine designated FLXLN2 was used. The input data to this particular routine are the wind speed at one level together with surface roughness length $z_{0}$ (the alternative version of wind speed at two levels is used to avoid $z_{0}$ ), air temperature $(T)$ and the cloud cover $(N)$. The solar elevation was calculated by an estimation scheme which used geographical position and time (Holstag and van Ulden 1983).

\section{Results and discussion}

The vertical wind profile calculated by using two levels of wind and temperature data are plotted and compared with observed data in figures 5-6. The comparison is reasonably good for all cases.

Values of $z_{0}$ calculated by the present approach are also shown in these figures. As the test region is open flat terrain, $z_{0}$ should be of the order of $0.03 \mathrm{~m}$ according to Davenport's terrain classification. The calculated values of $z_{0}$ lay in the range 0.01 to $0.03 \mathrm{~m}$. The variation in the values of $z_{0}$ was due to the fact that $z_{0}$ was azimuthally 

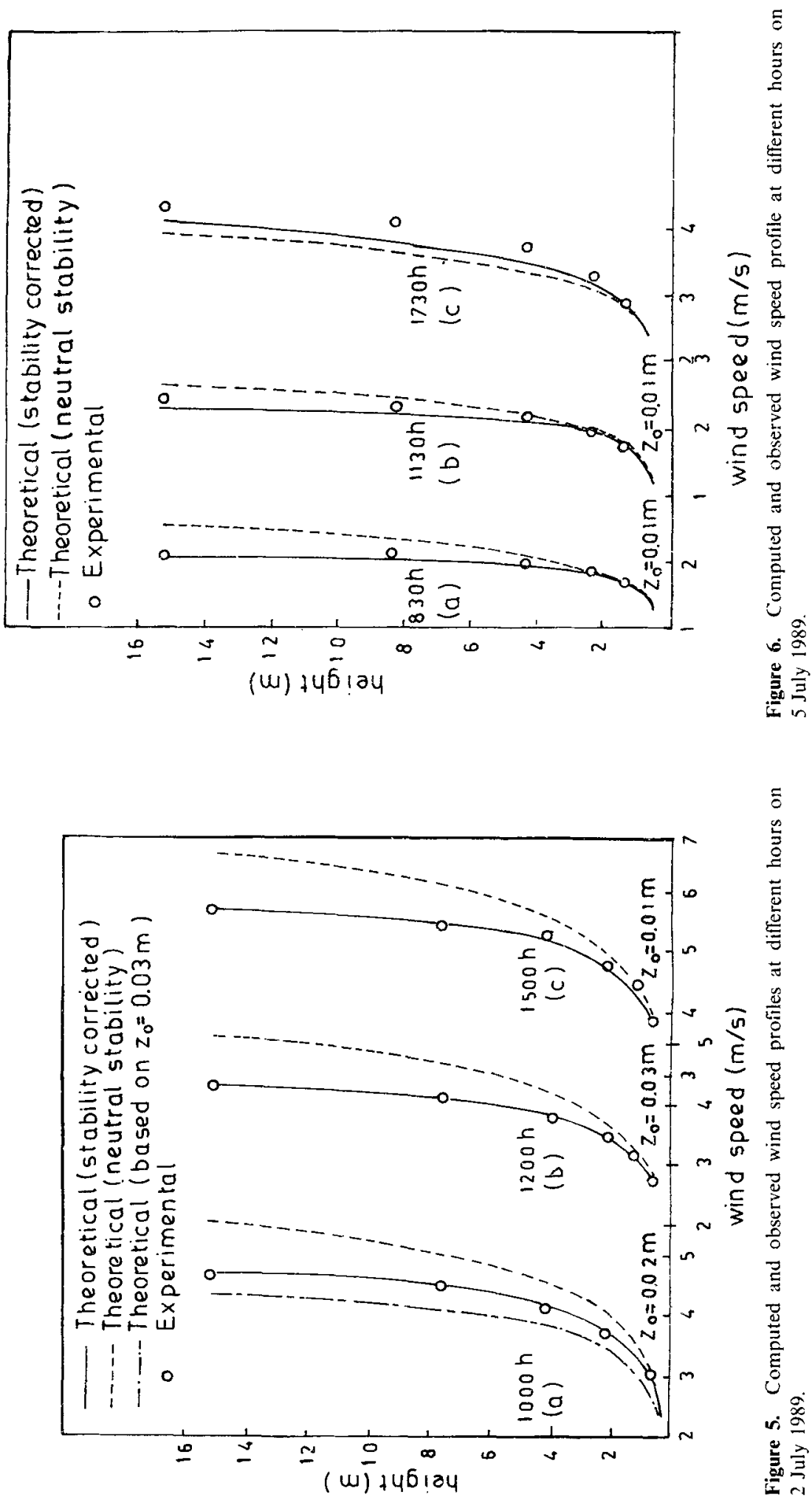
dependent, i.e. $z_{0}$ changed with wind direction which generally varied between west and south-west.

To illustrate the effects of stability of the surface layer, a wind speed profile was calculated by assuming the flow to be neutral (by putting $\psi_{M}(z / L)=\psi_{M}\left(z_{0} / L\right)=0$ in (2)). The difference between the stability corrected profile and the neutral profile was seen to be negligible at lower heights up to $2 \mathrm{~m}$, above which the effect of temperature stratification became significant.

The importance of calculating $z_{0}$ for determining the vertical wind profile is emphasised in figure 5a. The wind profile calculated from (2) by taking $z_{0}=0.03 \mathrm{~m}$ is shown in this figure. The difference in the two solutions based on calculated values of $z_{0}=0.02 \mathrm{~m}$ and an assumed value of $z_{0}=0.03 \mathrm{~m}$ is significant at all levels.

The results presented in figures 5-6 are for unstable air, except for the case shown in figure 6c. This for stable air $(L>0)$ when $z_{0}$ could not be computed, and the vertical wind profile was obtained by using (19) instead of (2).

To check the accuracy of surface roughness length, the computations were also by least squares. Calculated values of $z_{0}$ using this method are shown in figure 7 . The agreement between the present method and by least squares was reasonably good.

A comparison of the calculated surface roughness length by the two methods are shown in table 1 for 18 observations during the period 2 July to 5 July 1989 . For stable stratification $z_{0}$ could not be calculated by the present method, while least squares failed to provide a good estimate of $z_{0}$ when the scatter was high. The agreement between the two methods in most of the other cases was good.

To check the accuracy of the present evaluation of surface fluxes of momentum $(\tau)$

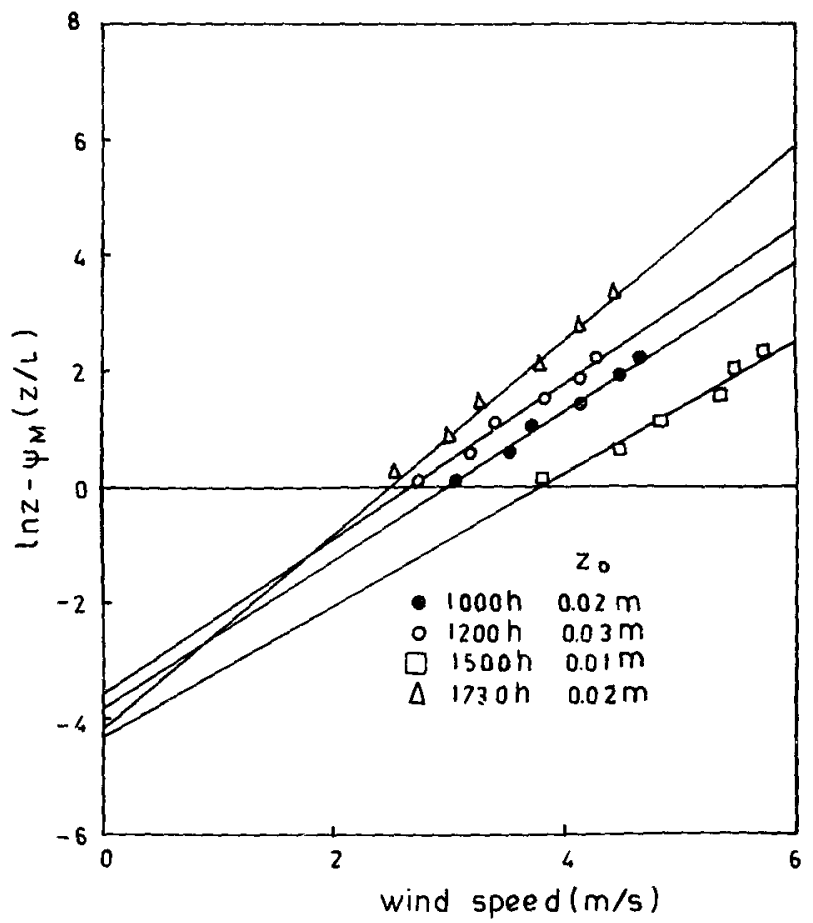

Figure 7. Wind speed against $\ln z-\psi_{M}(z / L)$ at different hours on 2 July 1989. 
Table 1. Comparison of computed surface roughness length $\left(z_{0}\right)$.

\begin{tabular}{llccc}
\hline $\begin{array}{l}\text { No. of } \\
\text { observations }\end{array}$ & Date & $\begin{array}{c}\text { Time } \\
\text { (I.S.T) }\end{array}$ & $\begin{array}{c}z_{0}(\mathrm{~m}) \\
\text { Present method }\end{array}$ & $\begin{array}{c}z_{0}(\mathrm{~m}) \\
\text { Least squares }\end{array}$ \\
\hline 1 & 2 July & 1000 & 0.02 & 0.02 \\
2 & 2 July & 1200 & 0.03 & 0.03 \\
3 & 2 July & 1500 & $0 \cdot 01$ & 0.01 \\
4 & 2 July & 1730 & - & 0.02 \\
5 & 3 July & 0530 & 0.04 & 0.06 \\
6 & 3 July & 0830 & 0.02 & 0.02 \\
7 & 3 July & 1130 & - & 0.00 \\
8 & 3 July & 1430 & $0 \cdot 02$ & 0.02 \\
9 & 3 July & 1730 & 0.51 & 0.70 \\
10 & 4 July & 0530 & - & - \\
11 & 4 July & 0830 & 0.01 & 0.01 \\
12 & 4 July & 1130 & 0.00 & 0.01 \\
13 & 4 July & 1430 & - & 0.00 \\
14 & 4 July & 1730 & - & 0.01 \\
15 & 5 July & 0830 & 0.01 & 0.01 \\
16 & 5 July & 1130 & 0.01 & 0.01 \\
17 & 5 July & 1730 & - & 0.00 \\
18 & 5 July & 2358 & 0.00 & - \\
\hline
\end{tabular}

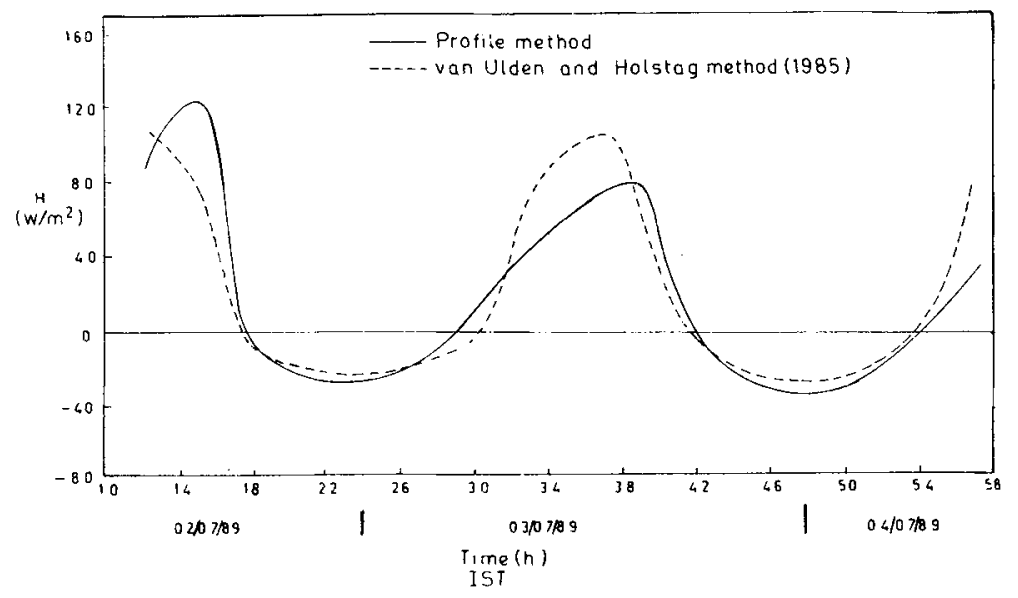

Figure 8. Variation of sensible heat flux with time over a 48 hour period.

and sensible heat $(H)$, the results were compared with those obtained by an energy budget (van Ulden and Holstag 1985) in figures 8-9. A comparison of the two solutions for sensible heat over a 48-hour period was good, except around 1200 hours. The peak value by energy budget was around 1200 hours, while by the present method it was at 1430 hours. The solution obtained by the present approach seems to be more consistent with the general observation (figure 3) that the warmest hour of the day is around 1430-1500 hours. A comparison of the momentum flux calculation in figure 9 is reasonably good over a 48 hour period. 


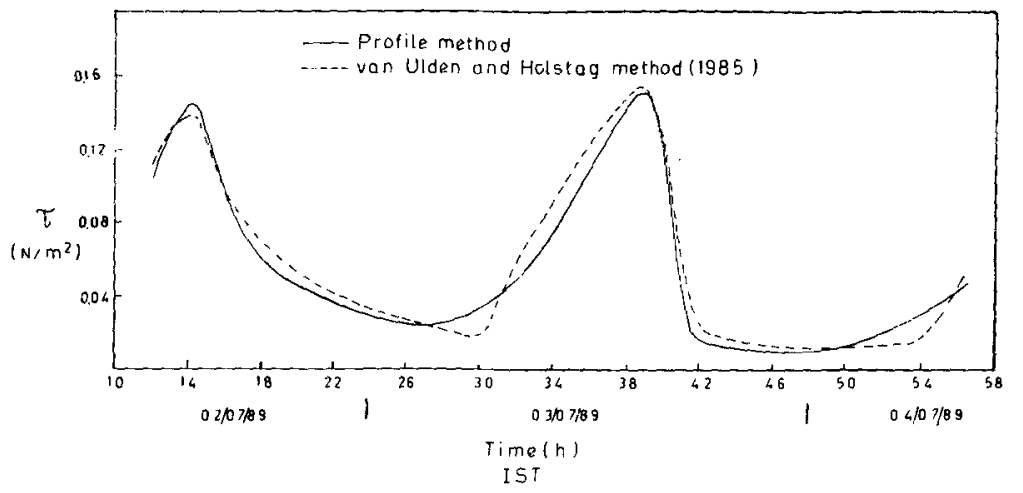

Figure 9. Variation of momentum flux with time over a 48 hour period.

\section{Conclusions}

A $30 \mathrm{~m}$ high tower, equipped with fast response sensors at six levels was installed at IIT, Kharagpur under the MONTBLEX program. Data were collected during the first week of July 1989. The tower data illustrate some of the atmospheric surface layer characteristics.

A profile method, using two levels for wind and temperature, was used together with a method of calculating surface roughness length. The theoretical results were compared with the tower data, as well as with the energy budget method of van Ulden and Holstag (1985). The vertical wind profiles calculated by the present method agrees well with tower observations, while the surface roughness length $\left(z_{0}\right)$ compares well with values obtained by least squares. Computed surface fluxes of momentum and sensible heat compare well with those obtained by the energy budget method.

\section{Acknowledgements}

The authors are grateful to the Department of Science and Technology for granting a fund to the MONTBLEX program. The authors are also grateful to all those involved in the planning and execution of MONTBLEX program. We would also like to thank Dr D K Nanda and Dr B K Singh of the Computer Centre for help in processing the tower data.

\section{References}

Berkowicz R and Prahm L P 1982 Evaluation of the profile method for estimation of surface fluxes of momentum and heat; Atmos. Environ. 16 2809-2819

Businger J A, Wyngaard J C, Izumi Y and Bradley E F 1971 Flux-profile relationships in the atmospheric surface layer; J. Atmos. Sci. $28181-189$

Davenport A G 1960 Rationale for determining design wind velocities; J. Struct. Div. Proc. Am. Soc. Civ. Eng. 86 39-68

Dyer A 11974 A review of flux-profile relationships; Bound. Laver Meteorol. 7 363-372

Holstag A A M 1984 Estimates of diabatic wind speed profiles from near surface weather observations; Bound. Layer Meteorol. $29225-250$ 
Holstag A A M and van Ulden A P 1983 A simple scheme for daytime estimates of the surface fluxes from routine weather data; J. Climate Appl. Meteorol. 22 517-529

McBean G A 1979 The planetary boundary layer; Tech. Note No. 165 WMO 530201 pp

Nieuwstadt F T M 1978 The computation of the friction velocity $u_{*}$ and the temperature $T_{*}$ from temperature $T_{*}$ and wind velocity profiles by least square methods: Bound. Layer Meteorol. 19 235-246

Paulson C A 1970 The mathematical representation of wind speed and temperature profiles in the unstable atmospheric surface layer; $J$. Appl. Meteorol. 9 856-861

van Ulden A P and Holstag A A M 1985 Estimation of atmospheric boundary layer parameters for diffusion applications; J. Climate Meteorol. 24 1196-1207

Wieringa J 1976 An objective exposure correction method for average wind speeds measured at a sheltered location; Q. J. R. Meteorol. Soc. London 102 241-253

Wieringa J 1980 Representativeness of wind observations at airports; Bull. Am. Meteorol. Soc. 61 962-971

Wieringa J 1983 Description requirements for assessment of non-ideal wind stations - for example Aachen; J. Wind Eng. Ind. Aerodyn. 11 111-121 